\title{
Riccati Differential Equation for Hypergeometric Differential Equation
}

\author{
TAKAHIRO NAKAGAWA
}

ABSTRACT - In this paper, we study the solutions of the Riccati differential equation collesponding to a $p$-adic differential equation which is solvable on the generic disc. As an application, we consider the Grothendieck conjecture for Riccati differential equations. We see that the Riccati differential equation for some globally nilpotent differential equation with coefficients in $Q(t)$ have, for almost all prime number, a solution in $\mathrm{F}_{p}(t)$, but do not have any algebraic solutions.

\section{Introduction}

Let $p$ be a prime and $K$ a complete non-archimedean valued field with mixed characteristics. In this paper, we consider the solutions of the Riccati differential equation for a $p$-adic differential equation $L$ which is solvable on the generic disc $D\left(t, 1^{-}\right.$) (see the Definition 2.5).

We attach the Riccati differential equation $C(L)_{1}$ for each linear ordinary differential operator $L$ in section 3. Let $E$ be the completion of $K(t)$ under the Gauss norm. We see that a solution of the Riccati differential equation $C(L)_{1}$ for $L$ on $E$ is connected with a bounded solution of $L$ at the generic point.

Let bddKer $\operatorname{Ke}_{t}(L)$ be the vector space consisting of the bounded solutions of $L$. If its dimension is one, then $C(L)_{1}$ has a unique solution on $E$. In particular if we can reduce $L$ modulo $p$, then $C(L)_{1}$ has a solution on the rational function field $\bar{K}(t)$ over the residue field $\bar{K}$ of $K$.

Let $L$ be an $n$-th order linear ordinary differential operator with coefficients in $\mathrm{Q}(t)$. The Grothendieck conjecture (in its simplest form) asserts the following statements are equivalent (see [Put]):

(i) the equation $L(y)=0$ has $n$ linear independent (over $\mathbb{Q}^{\text {alg }}$ ) solutions which are algebraic over $\mathrm{Q}(t)$.

(ii) For almost all $p$, the equation $L(y)=0$ has $n$ linear independent solutions over the field $\mathrm{F}_{p}\left(t^{p}\right)$ in the field $\mathrm{F}_{p}(t)$.

(*) Indirizzo dell'A.: Mathematical Institute CHIBA University, 263-8522 Japan.

E-mail: tnakagaw@g.math.s.chiba-u.ac.jp 
Van der Put studied the Grothendieck conjecture for non-linear differential equations. He proved that the differential equation $y^{\prime}=\left(1 / t^{2}\right) y+1$ has no algebraic solutions but there exists a solution in $\mathbb{F}_{p}(t)$ for all primes $p$. He also proved that the conjecture is true for some Risch equations [Put], [Put2].

We will prove the following theorem.

THEOREM 1.1. Let $L$ be a monic differential operator with coefficients in $Q(t)$. We assume that when we regard $L$ as a p-adic differential equation, $L$ is solvable on the generic disc $D(t, 1)$ for almost all prime $p$, and the dimension of the vector space which consists of the bounded solutions of $L$ is one. If $L$ has no algebraic solutions, then $C_{1}(L)$ modulo $p$ has a solution on $\mathbb{F}_{p}(t)$ for almost all prime $p$ and no algebraic solutions.

In particular some hypergeometric differential equations satisfy the condition of Theorem 1.1.

The author is grateful to Professor S. Matsuda for his helpful advice and thank referee for his suggestion.

\section{Notation}

\section{1 - Analytic elements}

Let $K$ be a complete non-archimedean valued field with mixed characteristics and $\bar{K}$ the residue field of $K$. Let $K(t)$ be the field of rational functions. For a polynomial $f=\sum a_{i} t^{i}$ with coefficients in $K$, we set

$$
|f|_{0}=\max \left|a_{i}\right| .
$$

The field of rational functions $K(t)$ has the Gauss norm $|*|_{0}$ defined by $|f|_{0}=|g|_{0} /|h|_{0}$ if $f=g / h$ and $g, h \in K[t]$. Let $E$ be a completion of $K(t)$ under the Gauss norm. An element of $E$ is called an analytic element. The derivation $d / d t$ of $K(t)$ is extended to $E$. We regard $(E, d / d t)$ as a differential field.

For $a \in E$ and $r \in \mathbb{R}_{>0}$, let

$$
D\left(a, r^{-}\right)=\left\{z \in E|| z-\left.a\right|_{0}<r\right\}
$$

and

$$
D(a, r)=\left\{z \in E|| z-\left.a\right|_{0} \leq r\right\} .
$$


Riccati Differential Equation for Hypergeometric Differential Equation 181

We define the ring of analytic functions on the disc $D\left(a, r^{-}\right)$to be $\mathcal{A}(a, r)=\left\{\sum_{n=0}^{\infty} a_{n}(x-a)^{n} \in E[[x-a]]\left|a_{n} \in E, \lim _{n \rightarrow \infty}\right| a_{n} \mid s^{n}=0\right.$ for all $\left.0 \leq s<r\right\}$.

We define the subring $E[[x-a]]_{0}$ of the ring of analytic functions $\mathcal{A}(a, 1)$ by

$$
E[[x-a]]_{0}=\left\{\sum_{n=0}^{\infty} a_{n}(x-a)^{n} \in E[[x-a]]\left|\sup _{0 \leq n<\infty}\right| a_{n} \mid<\infty\right\} .
$$

Proposition 2.1 ([Christol, 2.5.1]). We define a ring homomorphism $\tau: E \rightarrow E[[x-t]]_{0}$ so that, for $f \in E$,

$$
\tau: E \rightarrow E[[x-t]]_{0} f \mapsto \sum_{n=0}^{\infty} \frac{(d / d t)^{n}(f)}{n !}(x-t)^{n} .
$$

Then $\tau$ is an isometric isomorphism from $E$ to the subfield of $E[[x-t]]_{0}$ consisting of elements $f$ which satisfy:

$$
\tau((d / d t)(f))=(d / d x)(\tau(f))
$$

\section{2 - Differential modules}

Let $(H, D)$ be a differential field of characteristic zero and $C_{H}$ the constant field of $H$. We say differential field $\left(L, D^{\prime}\right)$ is a differential extension of $(H, D)$ if $L \supset H$ and $\left.D^{\prime}\right|_{H}=D$. An $H$-differential module $(M, \nabla)$ is a free $H$-module $M$ of finite type endowed with a $C_{H}$-linear homomorphism $\nabla(D)$ which satisfies $\nabla(D)(a m)=D a \cdot m+a \nabla(D)(m)$ for $a \in H$ and $m \in M$.

Let $(M, \nabla)$ be an $H$-differential module and $L$ a differential extension of $H$. An $H$-linear map $s: M \rightarrow L$ is said horizontal if $s(\nabla m)=D(s(m))$ for $m \in M$. We denote the $C_{H}$-vector space by $\operatorname{Hom}_{\nabla}(M, L):=$ $\left\{s \in \operatorname{Hom}_{C_{H}}(M, K) \mid s\right.$ is horizontal $\}$.

Proposition 2.2. Let $(M, \nabla)$ be an H-differential module. For any differential extension $L / H$,

$$
\operatorname{dim}_{C_{H}}\left(\operatorname{Hom}_{\nabla}(M, L)\right) \leq \operatorname{dim}_{H}(M)
$$


REMark 2.3. By the existence of Picard-Vessiot field of $H \otimes_{C_{H}} C_{H}^{a l g}$, there exists a differential extension $L / H$ such that equality (2.5) holds (see [PS]).

Proposition 2.4. Let $G$ be a matrix in $M_{n}(E)$. There exists a unique matrix $U$ of $G L_{n}(E[[x-t]])$ such that

$$
U(t)=I_{n}, \quad(d / d x)(U)=\tau(G) U .
$$

Let $\left\{G_{i}\right\}_{i \in \mathrm{N}}$ be the sequence of matrix in $M_{n}(E)$ defined by

$$
G_{0}=I_{n}, \quad G_{i+1}=(d / d t)\left(G_{i}\right)+G_{i} G .
$$

Then we have

$$
U=\sum_{i=0}^{\infty} G_{i} \frac{(x-t)^{i}}{i !}
$$

Definition 2.5. Let $(M, \nabla)$ be an $E$-differential module of rank $n$. Let $e_{1}, \cdots, e_{n}$ be a basis of $M$ and $G=\left(g_{i j}\right)$ the matrix such that $\nabla(D) e_{i}=\sum_{j=0}^{n} g_{i j} e_{j}$. We say $(M, \nabla)$ is solvable over $D(t, r)$ if the matrix $U$ which is defined by (2.6) is an element of $\mathrm{GL}_{n}(\mathcal{A}(t, r))$.

For a differential field $(H, D)$ which satisfies $K(t) \subset H \subset E$, we call an $H$-differential module $(M, \nabla)$ is solvable over $D(t, r)$ if the $E$-differential module $\left(M \otimes_{H} E, \nabla\right)$ is solvable over $D(t, r)$.

\section{Riccati differential equation}

Let $(H, D)$ be a differential field of characteristic zero and $C_{H}$ a constant field of $H$. Let $L=D^{n}-g_{n} D^{n-1}-\cdots-g_{1}$ be a differential operator with coefficients in $H$. Then $(M, \nabla)=H[D] / H[D] L$ is an $H$-differential module of rank $n$. Let $G$ be the matrix of representation of $\nabla(D)$ with respect to the basis $1, \cdots, D^{n-1}$ of $(M, \nabla)$, i.e,

$$
G=\left(\begin{array}{ccccc}
0 & 1 & 0 & \cdots & 0 \\
0 & 0 & 1 & \cdots & 0 \\
\vdots & & & & \\
0 & & & \cdots & 1 \\
g_{1} & g_{2} & & \cdots & g_{n}
\end{array}\right) .
$$


Riccati Differential Equation for Hypergeometric Differential Equation 183

We suppose that $L$ has a decomposition

$$
L=L_{1} \circ R, \quad L_{1}, R \in H[D] \text { and } R \text { is monic. }
$$

We write $R=D^{k}-c_{k} D^{k-1}-\cdots-c_{1}$. Since the canonical morphism $M=H[D] / H[D] L \rightarrow H[D] / H[D] R$ is surjective, the induced morphism $(H[D] / H[D] R)^{\vee} \rightarrow M^{\vee}$ is injective.

Let $f_{1}, \cdots, f_{n}$ (resp. $l_{1}, \cdots, l_{k}$ ) be the dual basis of the basis $1, D, \cdots, D^{n-1}$ of $M$ (resp. $\left.(H[D] / H[D] R)\right)$. If we regard $(H[D] / H[D] R)^{\vee}$ as a submodule of $M^{\vee}$ then each $l_{i}$ is a linear combination of $f_{i}$. In fact, by the definition of dual basis, we have

$$
\left(\begin{array}{c}
l_{1} \\
l_{2} \\
\vdots \\
l_{k}
\end{array}\right)=C\left(\begin{array}{c}
f_{1} \\
f_{2} \\
\vdots \\
f_{n}
\end{array}\right)
$$

such that $C=\left\{c_{i, j}\right\}$ is the $k \times n$ matrix defined by

$$
D^{m-1}=c_{k, m} D^{k-1}+c_{k-1, m} D^{k-2}+\cdots+c_{1, m} \quad(\bmod R) .
$$

In particular $c_{j, k+1}=c_{j}$ and if $j \leq k$ then $c_{i, j}=\delta_{i, j}$.

Let $F / H$ be a differential extension such that $\operatorname{dim}_{C_{H}}\left(\operatorname{Hom}_{\nabla}(M, F)\right)=$ $\operatorname{dim}_{H}(M)$ (see Remark 2.3). Let $s_{1}, \ldots, s_{n}$ be a basis of $\operatorname{Hom}_{\nabla}(M, F)$. By the equality (3.2), we have

$$
\left(\begin{array}{cccc}
s_{1}\left(l_{1}\right) & s_{2}\left(l_{1}\right) & \cdots & s_{n}\left(l_{1}\right) \\
s_{1}\left(l_{2}\right) & s_{2}\left(l_{2}\right) & \cdots & s_{n}\left(l_{2}\right) \\
\vdots & & & \vdots \\
s_{1}\left(l_{k}\right) & s_{2}\left(l_{k}\right) & \cdots & s_{n}\left(l_{k}\right)
\end{array}\right)=C\left(\begin{array}{cccc}
s_{1}\left(f_{1}\right) & s_{2}\left(f_{1}\right) & \cdots & s_{n}\left(f_{1}\right) \\
s_{1}\left(f_{2}\right) & s_{2}\left(f_{2}\right) & \cdots & s_{n}\left(f_{2}\right) \\
\vdots & & & \vdots \\
s_{1}\left(f_{n}\right) & s_{2}\left(f_{n}\right) & \cdots & s_{n}\left(f_{n}\right)
\end{array}\right) .
$$

Operating $D$ on both sides the equation (3.4), we have

$$
\begin{aligned}
& -\left(\begin{array}{ccc}
0 & \cdots & c_{1} \\
& & \vdots \\
& & \\
& 1 & c_{k}
\end{array}\right)\left(\begin{array}{cccc}
s_{1}\left(l_{1}\right) & s_{2}\left(l_{1}\right) & \cdots & s_{n}\left(l_{1}\right) \\
s_{1}\left(l_{2}\right) & s_{2}\left(l_{2}\right) & \cdots & s_{n}\left(l_{2}\right) \\
\vdots & & & \vdots \\
s_{1}\left(l_{k}\right) & s_{2}\left(l_{k}\right) & \cdots & s_{n}\left(l_{k}\right)
\end{array}\right) \\
& =\left(D(C)-C\left(\begin{array}{cccc}
0 & \cdots & & g_{1} \\
1 & \cdots & & g_{2} \\
\vdots & & \vdots & \\
0 & \cdots & 1 & g_{n}
\end{array}\right)\right)\left(\begin{array}{cccc}
s_{1}\left(f_{1}\right) & s_{2}\left(f_{1}\right) & \cdots & s_{n}\left(f_{1}\right) \\
s_{1}\left(f_{2}\right) & s_{2}\left(f_{2}\right) & \cdots & s_{n}\left(f_{2}\right) \\
\vdots & & & \vdots \\
s_{1}\left(f_{n}\right) & s_{2}\left(f_{n}\right) & \cdots & s_{n}\left(f_{n}\right)
\end{array}\right)
\end{aligned}
$$


Since the matrix $\left(s_{i}\left(f_{j}\right)\right)_{i, j}$ is invertible, we have

$$
-\left(\begin{array}{ccc}
0 & \cdots & c_{1} \\
1 & & \vdots \\
& & \\
& 1 & c_{k}
\end{array}\right) C=D(C)-C\left(\begin{array}{cccc}
0 & \cdots & & g_{1} \\
1 & \cdots & & g_{2} \\
\vdots & & \vdots & \\
0 & \cdots & 1 & g_{n}
\end{array}\right) .
$$

Therefore we have the equations

$$
\begin{gathered}
c_{1} c_{k, n}+D\left(c_{1, n}\right)-g_{1}-c_{1, k+1} g_{k+1}-\cdots-c_{1, n} g_{n}=0, \\
c_{1, n}+c_{2} c_{k, n}+D\left(c_{2, n}\right)-g_{2}-c_{2, k+1} g_{k+1}-\cdots-c_{2, n} g_{n}=0, \\
\vdots \\
c_{k-1, n}+c_{k} c_{k, n}+D\left(c_{k, n}\right)-g_{k}-c_{k, k+1} g_{k+1}-\cdots-c_{k, n} g_{n}=0 .
\end{gathered}
$$

We define the differential system $C(L)_{k}$ in $k$-variable $y_{1}, \ldots, y_{k}$ by

$$
\begin{gathered}
y_{1} y_{k, n}+D\left(y_{1, n}\right)-g_{1}-y_{1, k+1} g_{k+1}-\cdots-y_{1, n} g_{n}=0 \\
y_{1, n}+y_{2} y_{k, n}+D\left(y_{2, n}\right)-g_{2}-y_{2, k+1} g_{k+1}-\cdots-y_{2, n} g_{n}=0 \\
\vdots \\
y_{k-1, n}+y_{k} y_{k, n}+D\left(y_{k, n}\right)-g_{k}-y_{k, k+1} g_{k+1}-\cdots-y_{k, n} g_{n}=0
\end{gathered}
$$

where $y_{i, j}(1 \leq i \leq k, j \geq 1)$ is defined inductively by

$$
\begin{aligned}
& y_{1,1}=1, y_{j, 1}=0 \quad(2 \leq j \leq k), \\
& y_{1, m+1}=D\left(y_{1, m}\right)+y_{k, m} \cdot y_{1}, \quad y_{j, m+1}=D\left(y_{j, m}\right)+y_{k, m} \cdot y_{j}+y_{j-1, m} \quad(2 \leq j \leq k) .
\end{aligned}
$$

By definition, the vector $\left(c_{1}, \cdots, c_{k}\right)$ is solutions of differential system $C(L)_{k}$.

In particular, $C(L)_{1}$ is a differential equation

$$
y_{1} y_{1, n}+D\left(y_{1, n}\right)-g_{1}-y_{1,2} g_{2}-\cdots-y_{1, n} g_{n}=0
$$

where $y_{1, j}$ is defined inductively by

$$
y_{1,1}=1, \quad y_{1, m+1}=D\left(y_{1, m}\right)+y_{1, m} \cdot y_{1} .
$$

Conversely we assume differential system $C(L)_{k}$ has a solution $\left(c_{1}, \cdots, c_{k}\right) \in H^{k}$. Let $F / H$ be a differential extension which satisfies $\operatorname{dim}_{C_{H}}\left(\left\{y \in F \mid\left(D^{k}-c_{k} D^{k-1}-\cdots-c_{1}\right) y=0\right\}\right)=k$ (see Remark 2.3). If $y \in F$ satisfies the equality $\left(D^{k}-c_{k} D^{k-1}-\cdots-c_{1}\right) y=0$ then we have 
Riccati Differential Equation for Hypergeometric Differential Equation 185

$$
\begin{aligned}
& G\left(\begin{array}{c}
y \\
D y \\
\vdots \\
D^{n-1} y
\end{array}\right)=G \cdot{ }^{t} C\left(\begin{array}{c}
y \\
D y \\
\vdots \\
D^{k-1} y
\end{array}\right) \\
& =D\left({ }^{t} C\right)\left(\begin{array}{c}
y \\
D y \\
\vdots \\
D^{k-1} y
\end{array}\right)+{ }^{t} C \cdot\left(\begin{array}{ccccc}
0 & 1 & & \cdots & 0 \\
& 0 & 1 & & 0 \\
\vdots & & & & \\
c_{1} & c_{2} & & & c_{k}
\end{array}\right)\left(\begin{array}{c}
y \\
D y \\
\vdots \\
D^{k-1} y
\end{array}\right) \\
& =D\left({ }^{t} C\right)\left(\begin{array}{c}
y \\
D y \\
\vdots \\
D^{k-1} y
\end{array}\right)+{ }^{t} C \cdot\left(\begin{array}{c}
D y \\
D^{2} y \\
\vdots \\
D^{k} y
\end{array}\right)=\left(\begin{array}{c}
D y \\
D^{2} y \\
\vdots \\
D^{n} y
\end{array}\right) \text {. }
\end{aligned}
$$

Therefore $y$ satisfies a solution of differential equation

$$
D^{n} y=g_{n} D^{n-1} y+g_{n-1} D^{n-2} y+\cdots+g_{1} y
$$

By proposition 2.2, we have a decomposition

$$
D^{n}-g_{n} D^{n-1}-\cdots-g_{1} \in H[D]\left(D^{k}-c_{k} D^{k-1}-\cdots-c_{1}\right) .
$$

As a result, we have the following proposition.

Proposition 3.1. Let $H$ be a differential field such that the characteristic of $H$ is zero. Let $L=D^{n}-g_{n} D^{n-1}-\ldots-g_{1}$ be a differential polynomial with coefficients in $H$.

There is a one to one correspondence between a $k$-th order differential operator $R \in H[D]$ which satisfies $L \in H[D] R$ and a solution of differential system $C(L)_{k}$ in $H^{k}$.

If there exists a differential polynomial $R=D^{k}-c_{k} D^{k-1}-\cdots-c_{1}$ such that $L \in H[D] \cdot R$ then $\left(c_{1}, c_{2}, \ldots, c_{k}\right) \in H^{k}$ is a solution of $C(L)_{k}$. Conversely, if $\left(c_{1}, c_{2}, \ldots, c_{k}\right) \in H^{k}$ is a solution of $C(L)_{k}$ then the differential polynomial $R=D^{k}-c_{k} D^{k-1}-\cdots-c_{1}$ satisfies $L \in H[D] \cdot R$.

By induction, we have the following lemma.

Lemma 3.2. We assume $H=E$. Let $L=D^{n}-g_{n} D^{n-1}-\ldots-g_{1}$ be a differential polynomial with coefficients in $E$. If $\left|g_{j}\right|_{0} \leq 1$ for $j=1, \cdots, n$, then, for any solution $y \in E$ of $C_{1}(L)$, we have $|y|_{0} \leq 1$. 
EXAMPLE 3.3. For a differential operator $L=D^{2}-g_{2} D-g_{1}$ of rank 2 , $C(L)_{1}$ is a Riccati differential equation $c^{2}+c^{\prime}-g_{1}-g_{2} c=0$. Young considered in this case [Young].

\section{Analytic element solution of Riccati differential equation}

Let $L$ be a differential operator with coefficients in $E$ and $D=d / d t$. Let $C(L)_{k}$ be the differential system defined by $L$. We suppose that $L$ is solvable on $D(t, 1)$ (i.e., $E[D] / E[D] L$ is solvable on $D(t, 1)$ ). We denote the vector space which is generated by the solutions of (resp. the bounded solutions) of $L$ by

$$
\begin{aligned}
& \operatorname{Ker}_{t}(L)=\{y \in \mathcal{A}(t, 1) \mid \tau(L) y=0\}, \\
& \left(\operatorname{resp}_{\operatorname{Kerbdd}}(L)=\left\{y \in E[[x-t]]_{0} \mid \tau(L) y=0\right\}\right) .
\end{aligned}
$$

In general, since $L$ is solvable on $D(t, 1)$, we have $\operatorname{dim}_{K}\left(\operatorname{Kerbdd}_{t}(L)\right) \geq 1$ by [Robba, Theorem 3.5]. If $\operatorname{dim}_{K}\left(\operatorname{Kerbdd}_{t}(L)\right) \leq n-1$, then there exists a monic differential operator $R \in E[D]$ such that $L \in E[D] R$ and $\operatorname{Ker}_{t}(R)=$ $\operatorname{Kerbdd}_{t}(L)$ by [Robba, Theorem 2.6].

Proposition 4.1. Let $L \in E[D]$ be a monic n-th order differential operator which is solvable on $D(t, 1)$.

(i) If $k=\operatorname{dim}_{K}\left(\operatorname{Kerbdd}{ }_{t}(L)\right) \leq n-1$, then $C(L)_{k}$ has a solution on $E$.

(ii) If $1=\operatorname{dim}_{K}\left(\operatorname{Kerbdd}_{t}(L)\right), C(L)_{1}$ has a unique solution on $E$.

(iii) If there exists fundamental system of solutions $y_{1}, \ldots y_{n}$ of $L$ at $t$ such that $y_{i}$ is exactly of log-growth $i-1$ (see the definition of log-growth in [C-T]) then, for each $j, C(L)_{j}$ has a unique solution over $E$.

Proof. There exists a monic differential operator $R \in E[D]$ such that $L \in E[D] R$ and $\operatorname{Ker}_{t}(R)=\{y \in E[[x-t]] \mid \tau(L) y=0$ and $y$ is log-growth $i\}$ by [Robba, Theorem 2.6]. By Proposition 3.1, there exists a solution of $C(L)_{k}$ on $E$ where $k=\operatorname{deg}(R)$. The uniqueness of case (ii),(iii) is confirmed by [Dwork1, Theorem 1].

By [D-R, Theorem 2.4.3 (iii)], we have the following proposition.

Proposition 4.2. Let $L \in E[D]$ be a monic n-th differential operator which is solvable on $D(t, 1)$. Assume $k=\operatorname{dim}_{K}\left(\operatorname{Kerbdd}_{t}(L)\right) \leq n-1$. Let $S$ be the set $\left\{\bar{a} \in \bar{K} \mid L\right.$ has no pole on $\left.D\left(a, 1^{-}\right)\right\}$. Then there exists a solution 
Riccati Differential Equation for Hypergeometric Differential Equation 187

$\left(y_{1}, \ldots, y_{k}\right) \in E^{k}$ of $C(L)_{k}$ such that

$$
\begin{aligned}
& \left\{\bar{a} \in S \mid \operatorname{dim}\left(\operatorname{Kerbdd}_{t}(L)\right)>\operatorname{dim}\left(\operatorname{Kerbdd}_{a}(L)\right)\right\} \subseteq \\
& \left\{\bar{a} \in S \mid y_{i} \text { is not analytic on } D\left(a, 1^{-}\right) \text {for some } i\right\}
\end{aligned}
$$

\section{Hypergeometric differential equation and Riccati differential equation $\bmod p$}

Let $p$ be a prime number and $D=d / d x$. For $a, b, c \in \mathbb{Q} \cap Z_{p}$, we set the hypergeometric differential operator

$$
L(a, b, c)=D^{2}+\frac{c-(1+a+b) x}{x(1-x)} D-\frac{a b}{x(1-x)} .
$$

We assume the following condition:

$$
\begin{aligned}
& c-a, c-b, b, a \text { all lie outside } Z_{s} . \\
& c \in \mathbb{Q}-\{0,-1,-2, \ldots\} .
\end{aligned}
$$

By [Dwork2, p. 11, Remark], we see the Riccati differential equation

$$
y^{2}+y^{\prime}+\frac{c-(1+a+b) x}{x(1-x)} y-\frac{a b}{x(1-x)}=0
$$

has no rational solutions.

For a rational number $a \in \mathbb{Q} \cap Z_{p}$, we set $\mu_{a} \in[0, p-1]$ such that $\mu_{a}=-a(\bmod p)$. According to Dwork, we say $(a, b, c) \in \mathbb{Q}$ is split (resp. unsplit) if $\mu_{c}<\min \left\{\mu_{a}, \mu_{b}\right\}$ or $\mu_{c}>\max \left\{\mu_{a}, \mu_{b}\right\}$ (resp. $\mu_{a}<\mu_{c}<\mu_{b}$ or $\left.\mu_{b}<\mu_{c}<\mu_{a}\right)$. There exists an integer $m$ such that

$$
F:=p^{m} \sum_{n=0}^{p-1} \frac{(a, n)(b, n)}{(c, n) n !} x^{n} \in Z_{p}[x]-p Z_{p}[x],
$$

where $(a, n)$ is defined

$$
(a, n)=\left\{\begin{array}{l}
1(n=0) \\
a(a+1) \cdots(a+n-1) \quad(n \geq 1) .
\end{array}\right.
$$

If $(a, b, c)$ is split, then $F$ is uniquely determined. Since $F$ is a solution of hypergeometric differential equation

$$
\left(D^{2}+\frac{c-(1+a+b) x}{x(1-x)} D-\frac{a b}{x(1-x)}\right) y=0(\bmod p)
$$


by Lemma 5.2, $F^{\prime} / F$ is a solution of the Riccati differential equation

$$
y^{2}+y^{\prime}+\frac{c-(1+a+b) x}{x(1-x)} y-\frac{a b}{x(1-x)}=0 \quad(\bmod p) .
$$

Under the special condition, Dwork proved that if $(a, b, c)$ is split, then $L(a, b, c)$ has a unbounded solution and if $(a, b, c)$ is unsplit, then $L(a, b, c)$ has two independent bounded solutions (see [Dwork2, Theorem 6.6, 9.4]). We consider solutions of the Riccati differential equation (5.7).

LEMma 5.1. We assume that $(a, b, c) \in\left(\mathrm{Q} \cap \mathrm{Z}_{p}\right)^{3}$ is unsplit. Then the Riccati differential equation (5.7) has infinite solutions.

PRoof. In this case, the hypergeometric differential equation (5.6) has solution

$$
u \sum_{n=0}^{\min \left\{\mu_{a}, \mu_{b}\right\}} \frac{(a, n)(b, n)}{(c, n) n !} x^{n}+v p^{m} \sum_{n=\mu_{c}+1}^{\max \left\{\mu_{a}, \mu_{b}\right\}} \frac{(a, n)(b, n)}{(c, n) n !} x^{n} \quad\left(u, v \in \mathbb{F}_{p}\left(x^{p}\right)\right)
$$

with $m \in Z_{\text {w }}$ wich satisfies $p^{m} \sum_{n=\mu_{c}+1}^{\max \left\{\mu_{a}, \mu_{b}\right\}} \frac{(a, n)(b, n)}{(c, n) n !} x^{n} \in Z_{p}[x]-p Z_{p}[x]$. By Lemma 5.2, the Riccati differential equation (5.7) has infinite solutions.

LEMmA 5.2. Let $R=D^{n}-g_{n} D^{n-1}-g_{n-1} D^{n-2}-\ldots-g_{1}$ be a differential operator with coefficients $\mathbb{F}_{p}(x)$. Then $R$ has a decomposition $R=Q \cdot(D-c)$ where $Q \in \mathbb{F}_{p}(x)[D]$ and $c \in \mathbb{F}_{p}(x)$ if and only if $c \in \mathbb{F}_{p}(x)$ is a solution of the Riccati differential equation $C(R)_{1}$.

Proof. Assume that $R=\left(D^{n-1}+b_{n-1} D^{n-2}+\cdots+b_{1}\right)(D-c)$ where $b_{1}, \cdots, b_{n-1}, c \in \mathbb{F}_{p}(x)$. Comparing the both sides, we have the equalities

$$
\begin{aligned}
g_{n} & =c-b_{n-1} \\
g_{n-1} & =\left(\begin{array}{c}
n-1 \\
1
\end{array}\right) c^{(1)}+c b_{n-1}-b_{n-2} \\
\vdots & \\
g_{i} & =\left(\begin{array}{c}
n-1 \\
n-i
\end{array}\right) c^{(n-i)}+\left(\begin{array}{c}
n-2 \\
n-i-1
\end{array}\right) c^{(n-i-1)} b_{n-1}+\cdots+c^{\prime}\left(\begin{array}{c}
i \\
1
\end{array}\right) b_{i+1}+c b_{i}-b_{i-1} \\
\vdots & \\
g_{2} & =\left(\begin{array}{c}
n-1 \\
n-2
\end{array}\right) c^{(n-2)}+\left(\begin{array}{c}
n-2 \\
n-3
\end{array}\right) c^{(n-3)} b_{n-1}+\cdots+c^{\prime}\left(\begin{array}{l}
2 \\
1
\end{array}\right) b_{3}+c b_{2}-b_{1} \\
g_{1} & =c^{(n-1)}+c^{(n-2)} b_{n-1}+\cdots+c^{(1)} b_{2}+c b_{1} .
\end{aligned}
$$


Riccati Differential Equation for Hypergeometric Differential Equation 189

We set $c_{m}, C_{m}$ by

$$
\begin{aligned}
& c_{m+1}=c_{m}^{\prime}+c c_{m}, \quad c_{1}=1 \\
& C_{m}=\sum_{i=1}^{m}\left(\begin{array}{c}
m-1 \\
i-1
\end{array}\right) c_{m-i+1} c^{(i-1)}-c_{m+1} .
\end{aligned}
$$

We have the equality

$$
-g_{1}-c_{2} g_{2}-\cdots-c_{n} g_{n}=c_{n+1}+C_{n}+\sum_{i=1}^{n-1} C_{i} b_{i} .
$$

To prove that $c$ is a solution of $C(R)_{1}$, we have only to prove $C_{m}=0$ for all $m$. We use induction on $m$. For $m=1, C_{1}=c_{1} c^{(0)}-c_{2}=0$. Therefore the case $m=1$ is true.

We assume that the assertion is true for $m=k-1$.

$$
\begin{aligned}
c_{k}^{\prime} & =\left(\sum_{i=1}^{k-1}\left(\begin{array}{c}
k-2 \\
i-1
\end{array}\right) c_{k-i} c^{(i-1)}\right)^{\prime} \\
& =\sum_{i=1}^{k-1}\left(\begin{array}{c}
k-2 \\
i-1
\end{array}\right)\left(c_{k-i}^{\prime} c^{(i-1)}+c_{k-i} c^{(i)}\right) \\
& =\sum_{i=1}^{k-1}\left(\begin{array}{c}
k-2 \\
i-1
\end{array}\right)\left(\left(c_{k-i+1}-c c_{k-i}\right) c^{(i-1)}+c_{k-i} c^{(i)}\right) \\
& =\sum_{i=1}^{k-1}\left(\begin{array}{c}
k-2 \\
i-1
\end{array}\right)\left(c_{k-i+1} c^{(i-1)}+c_{k-i} c^{(i)}\right)-c c_{k} \\
& =\sum_{i=2}^{k-1}\left(\left(\begin{array}{c}
k-2 \\
i-1
\end{array}\right)+\left(\begin{array}{c}
k-2 \\
i-2
\end{array}\right)\right) c_{k-i+1} c^{(i-1)}+c^{(k-1)} \\
& =\sum_{i=2}^{k-1}\left(\begin{array}{c}
k-1 \\
i-1
\end{array}\right) c_{k-i+1} c^{(i-1)}+c^{(k-1)}=C_{k}-c c_{k}+c_{k+1}
\end{aligned}
$$

This completes the induction.

Conversely let $c \in \mathbb{F}_{p}(x)$ be a solution of $C(R)_{1}$. We define $b_{1}, \ldots, b_{n-1} \in \mathbb{F}_{p}(x)$ as above. Then we have the decomposition $R=\left(D^{n-1}+b_{n-1} D^{n-2}+\cdots+b_{1}\right)(D-c)$. 
LEMMA 5.3. We assume that $(a, b, c) \in\left(\mathbb{Q} \cap Z_{p}\right)^{3}$ is split. The Riccati differential equation (5.7) has a unique solution if and only if

$$
\left(\frac{x^{\mu_{c}}(1-x)^{-1+\mu_{a}+\mu_{b}-\mu_{c}}}{F^{2}}\right)^{(p-1)} \neq 0(\bmod p)
$$

Proof. Substituting $y=z+F^{\prime} / F$ for $y$ in the Riccati differential equation (5.7), we have

$$
z^{2}+z^{\prime}+\left(2 \frac{F^{\prime}}{F}+\frac{c-(a+b+1) x}{x(1-x)}\right) z=0(\bmod p) .
$$

Substituting $z=h x^{\mu_{c}}(1-x)^{-1+\mu_{a}+\mu_{b}-\mu_{c}} / F^{2}$ for $z$ in the Riccati differential equation (5.7), we have

$$
x^{\mu_{c}}(1-x)^{-1+\mu_{a}+\mu_{b}-\mu_{c}} \frac{h^{2}}{F^{2}}+h^{\prime}=0(\bmod p) .
$$

We suppose $h \neq 0$. By this equality,

$$
\left(\frac{1}{h}\right)^{\prime}=\frac{x^{\mu_{c}}(1-x)^{-1+\mu_{a}+\mu_{b}-\mu_{c}}}{F^{2}}(\bmod p) .
$$

The differential equation (5.12) has a solution if and only if

$$
\frac{x^{\mu_{c}}(1-x)^{-1+\mu_{a}+\mu_{b}-\mu_{c}}}{F^{2}} \in \mathbb{F}_{p}\left(x^{p}\right) \oplus x \mathbb{F}_{p}\left(x^{p}\right) \oplus \ldots \oplus x^{p-2} \mathbb{F}_{p}\left(x^{p}\right)
$$

i.e.

$$
\left(\frac{x^{\mu_{c}(1-x)^{-1+\mu_{a}+\mu_{b}-\mu_{c}}}}{F^{2}}\right)^{(p-1)}=0(\bmod p)
$$

Proposition 5.4. We assume that $\mu_{c}=p-1$ and $(a, b, c)$ is split. Then the Riccati differential equation (5.7) has a unique solution $F^{\prime} / F$.

Proof. Let $v_{x}$ be a valuation of $\mathrm{F}_{p}(x)$ with respect to a prime ideal $x \mathbb{F}[x]$ of $\mathbb{F}[x]$. Since $p-1=\mu_{c}>\max \left\{\mu_{a}, \mu_{b}\right\}$ by assumption, $v_{x}(F)=0$. Therefore we have

$$
v_{x}\left(\left(\frac{1}{h}\right)^{\prime}\right)=v_{x}\left(\frac{x^{\mu_{c}}(1-x)^{-1+\mu_{a}+\mu_{b}-\mu_{c}}}{F^{2}}\right)=p-1 .
$$

Since there exists no $h \in \mathbb{F}(x)$ which satisfies (5.15), we have $h=0$. 
Riccati Differential Equation for Hypergeometric Differential Equation 191

\section{Algebraic solution of Riccati differential equation}

Let $L$ be a monic differential operator with coefficients in $Q(t)$ and $D=d / d t$. For almost all prime $p$, we can reduce $L$ modulo $p$. If we can reduce $L$ modulo $p$, then we denote $L$ modulo $p$ by $L_{p}$.

THEOREM 6.1. (i) If $L$ has an algebraic solution, then $C(L)_{1}$ has an algebraic solution.

(ii) We assume that for almost all prime $p, L$ is solvable on the generic disc $D(t, 1)$ as a p-adic differential equation. If $C(L)_{1}$ has a rational solution then $L$ has an algebraic solution.

(iii) We assume that for almost all prime $p, L$ is solvable on the generic disc $D(t, 1)$ and $\operatorname{dim}\left(\operatorname{bddKer}_{t}(L)\right)=1$ as a p-adic differential equation. Then for almost all prime $p, C(L)_{1}$ has a solution in $\mathrm{F}_{p}(t)$. And if $C(L)_{1}$ has an algebraic solution, then $L$ has an algebraic solution.

Proof. If $L$ has an algebraic solution then there exists an algebraic function $h$ such that $L \in Q(t)^{\operatorname{alg}}[D](D-h)$. Hence the Proposition 3.1 implies (i).

Suppose that $C(L)_{1}$ has a rational solution y. By Proposition 3.1, we have $L \in \mathbb{Q}(t)[D](D-y)$. Let $p$ be a prime such that when we regard $L$ as a $p$-adic differential equation, $L$ is solvable on the generic disc $D(t, 1)$ and that we can reduce $L$ and $D-y$ modulo $p$. By [DGS, 3. Proposition 5.1], $L_{p}$ is nilpotent. And by [DGS, 3. Theorem 2.1], $(D-y)_{p}$ is also nilpotent and therefore has a solution of $\mathbb{F}_{p}(t)$. Since for all almost prime $p,(D-y)_{p}$ has a solution of $\mathbb{F}_{p}(t)$, by Honda's theorem([Put2, Proposition 2.2]), $D-y$ has an algebraic solution. This implies (ii).

We will prove (iii). By Lemma 3.2 and Proposition 4.1(ii), for all almost prime $p, C(L)_{1}$ has a solution on $\mathrm{F}_{p}(t)$. We assume that $C(L)_{1}$ has an algebraic solution $y$ which is not a rational function. Let $G$ be the Galois group which corresponds to a finite Galois extension of $Q(t)$ contains $y$. For all $\sigma \in G, \sigma(y)$ is a solution of $C(L)_{1}$. Since $y$ is not a rational function, $C(L)_{1}$ has at least two algebraic solutions. By Proposition 3.1, this implies $\operatorname{dim}\left(\operatorname{bddKer}_{t}(L)\right)>1$. This is a contradiction.

Corollary 6.2. Let $L$ be a monic differential operator with coefficients in $\mathrm{Q}(t)$. We assume that for almost all prime $p$, when we regard $L$ as a p-adic differential equation, $L$ is solvable on the generic disc $D(t, 1)$ and $\operatorname{dim}\left(\operatorname{bddKer}_{t}(L)\right)=1$. If $L$ has no algebraic solutions, then $C(L)_{1}$ has a solution on $\mathrm{F}_{p}(t)$ for almost all prime $p$ and no algebraic solutions. 


\section{REFERENCES}

[Andre] Y. ANDRÉ, Dwork's conjecture on the logarithmic growth of solutions of p-adic differential equations, Compos. Math., 144 (2008), pp. 484-494.

[Baldassarri] F. BALDASSARRI, Continuity of the radius of convergence of differential equations on p-adic analytic curves, Invent. Math., 182, (3) (2010), pp. 513-584.

[C-T] B. CHIARELLOTTO - N. TsuzUKI, Logarithmic growth and Frobenius filtrations for solutions of p-adic differential equations, J. Inst. Math. Jussieu, 8 (2009), pp. 465-505.

[Christol] G. Christol, Modules différentiels et équations différentielles $p$ adiques, Queen's Papers in Pure and Appl. Math. 66, Queen's University, Kingston, ON, 1983.

[Dwork1] B. M. Dwork, On p-adic differential equations II, Annals of Math., 98, (2) (1973), pp. 366-376.

[Dwork2] B. M. Dwonk, Lectures on p-adic differential equations, Grundlehren Math. Wiss. 253, Springer- Verlag, New York-Berlin, 1982.

[DGS] B. Dwork - G. Gerotto - F. Sullivan, An Introduction to GFunctions, Annals of Math. Studies 133, Princeton University Press, Princeton, 1994.

[D-R] B. M. Dwork - P. RoBBa, On Ordinary Linear p-Adic Differential Equations, T.A.M.S., 231 (1977), pp. 1-46.

[Kedlaya] K. Kedlaya, p-adic differential equations, Cambridge University Press, Cambridge (2010).

[Put] M. VAN DER PUT, Reduction modulo $p$ of differential equations. Indag. Math. N. S., 7, (3) (1996), pp. 367-387.

[Put2] M. VAN DER PUT, Grothendieck's conjecture for the Risch equation $y^{\prime}=a y+b$. Indag. Math. (N.S.), 12 (2001), pp. 113-124.

[Put3] M. vaN DER PUT, Bounded p-adic differential equations Circumspice, Nijmegen, 2001.

[PS] M. VAN DER PUT - M. SInger, Galois theory of linear differential equations. Grundlehren der Mathematischen Wissenschaften 328. Springer 2003.

[R-C] P. RoBBA - G. CHRIstol, Équation différentielles p-adiques. Application aux sommes exponentielles. Actualités Math., Hermann, Paris, 1994.

[Robba] P. RoBba, On the index of p-adic differential operators I, Ann. of Math. (2), 101 (1975), pp. 280-316.

[Young] P. Young, Radii of p-adic convergence of generic solutions of homogeneous linear differential equations. Thesis (Ph.D.)-Oklahoma State University (1988), pp. 71.

Manoscritto pervenuto in redazione il 20 maggio 2011. 